\title{
Non-inferiority of The Cementless Total TKA Compared to The Cemented TKA, A m-Metanalysis
}

Tommaso Bonanzinga ${ }^{1,2},[\mathrm{MD}]$

ORCID: 0000-0003-2861-4699

Francesco Manlio Gambaro², [MD]

ORCID: 0000-0002-0691-0369

Riccardo Garibaldi ${ }^{3}$, [MD]

ORCID: 0000-0001-6380-2236

Federico Adravanti ${ }^{2}$, [MD]

ORCID: 0000-0001-5805-8617

Gerardo Fusco ${ }^{1,2}$, [MD]

ORCID: 0000-0002-9318-6523

Maurilio Marcacci ${ }^{1,2},[\mathrm{MD}]$

ORCID: 0000-0002-7484-1246

'IRCCS Istituto Clinico Humanitas, Via A. Manzoni 56, 20089 Rozzano, Milan, Italy.

${ }^{2}$ Humanitas University, Via Rita Levi Montalcini 4, 20090 Pieve Emanuele, Milan, Italy.

${ }^{3}$ Department of Surgery and Orthopedics, Regional Hospital of Locarno, Switzerland.

Corresponding Author: Tommaso Bonanzinga IRCCS Istituto Clinico Humanitas, Via A. Manzoni 56, 20089 Rozzano, Milan, Italy.

Humanitas University, Via Rita Levi Montalcini 4, 20090

Pieve Emanuele, Milan, Italy.

E-mail: t.bonanzinga@gmail.com

https://doi.org/10.32552/2021.ActaMedica.564

\section{nee) ABSTRACT Con}

Objective: Cemented total knee arthroplasty still represents the reference standard in the field of prothesis knee replacement; but since cementless total knee arthroplasties were introduced there have been strong discussions over the years among cemented and non-cemented total knee arthroplasties to establish which gives the best benefits for the patient and for the surgeon.

The purpose of this meta-analysis is to systematically analyze the use of cemented and cementless total knee arthroplasties by investigating clinical and radiological outcomes and rate of complications, in order to assess which techniques confers more benefits to the patient and the surgeon.

Materials and Methods: The current systematic review has been written in accordance to the Cochrane handbook and the PRISMA statement for reporting of systematic reviews incorporating network.

Results: Six randomized controlled trials were finally included in this systematic review. The statistical analysis revealed no significant differences in all clinical scores of interest (Knee Society Score, clinical and functional, Oxford Knee Score, Visual Analogue Score) and a similar revision rate.

Conclusion: the results of the current metanalysis suggest the noninferiority of cementless fixation with respect to cemented total knee arthroplasties in terms of clinical outcomes and survival rates of the implants.

Keywords: TKA, cemented, cementless, outcomes

Received: 4 July 2020, Accepted: 2 November 2021,

Published online: 1 November 2021 


\section{INTRODUCTION}

Nowadays the useof kneereplacementasa definitive osteoarthritis treatment is employed in younger patients compared to the past. Indeed, in the year 2011 the percentage of under 65 years patients undergoing total knee arthroplasty (TKA) has reached $50 \%$ and is expected to exceed $55 \%$ by the year 2030 [1]. With a gradually younger population undergoing TKAs, more functional outcomes and greater quality of life will be required by the patients; concomitantly an increased implant life will be needed. Cemented TKAs have been and are widely used in patients and still represent reference standard for TKA; but since cementless TKAs were introduced there have been strong discussions over the years among cemented and non-cemented TKAs to establish which gives the best benefits for the patient and for the surgeon [2,3]. Cementless TKA was not accepted widely because of problems encountered in long term follow of first implants, not showing any benefits with respect to classical cemented TKAs [4]. However, in the last decade, due to the development of new implant materials, interest in cementless TKAs has risen, settling ever more the debate between the use of cementless or cemented implants. The introduction of trabecular metal, a porous biomaterial resembling morphologically and biomechanically the patient trabecular bone, could minimize the risk of aseptic loosening of cementless TKA which represented the major cause of failure of primary TKA $[3,5]$. This hypothesis is strengthened by several studies that, with the use of radio stereometric analyses, demonstrated excellent fixation of the tibial component with only mild failure rate among non-modular tibial trays [6-7]. Other theoretical advantages of cementless TKA could include preservation of bone stock and elimination of complication related to the use of cement, such as retaining of loose cement fragments or third body wear. Another consideration that can be made regarding cementless fixation is the reduction of operative time because there is no need to wait for the cement curing. This leads to important benefits for the patient and surgeon reducing the risk of prosthetic joint infection (PJI) and surgical site infection, reducing the need of pneumatic ischemia because there is no need of complete exposure of trabecular bone when waiting for the cement hardening. Few systematic reviews and metaanalyses have compared radiological and clinical outcomes of cemented versus non-cemented TKAs and it's still unknown whether the cementless TKA could represent a valid alternative in knee replacement surgery or not. The purpose of this meta-analysis is to systematically analyze the use of cemented and cementless TKAs and to evaluate clinical and radiological results using validated scoring instruments, radiographic findings, rate of complications and survivorship rates.

\section{MATERIALS and METHODS}

The current systematic review has been written in accordance to the Cochrane handbook [8] and the PRISMA statement for reporting of systematic reviews incorporating network [9], of which the chart flow is summarized in Figure 1.

\section{Data Source}

A literature research was carried out by two independent authors (F.M.G. and T. B.) through September 2020 on PubMed, Google Scholar and Scopus databases with the following items: total knee arthroplasty, cemented, cementless. The research was limited to randomized controlled trials (RCT) conducted on human subjects. Moreover, in order to identify all eligible studies, we searched the Cochrane Library, the International Clinical Trials Registry (ICTRP) of the WHO, ClinicalTrials.gov and the EU Clinical Trials Register.

\section{Study Selection}

All the 513 articles obtained by the literature search were screened for relevance, and after careful reading of the abstract the full text of the relevant articles was analyzed before final inclusion. The last research was performed on October 1st, 2020. Based on the Oxford Center of Evidence-BasedMedicine only level I articles were found by the authors and included in the study. The inclusion criteria used to judge the relevance of an article for the current study were: RCT comparing TKA with neither components (femoral and tibial) cemented and TKA with both components cemented. For the study selection the following exclusion criteria have been applied: presence of hybrid prothesis (only one component cemented), studies including protheses implanted before the year 2000, follow up less than 2 years. 
PRISMA 2009 Flow Diagram
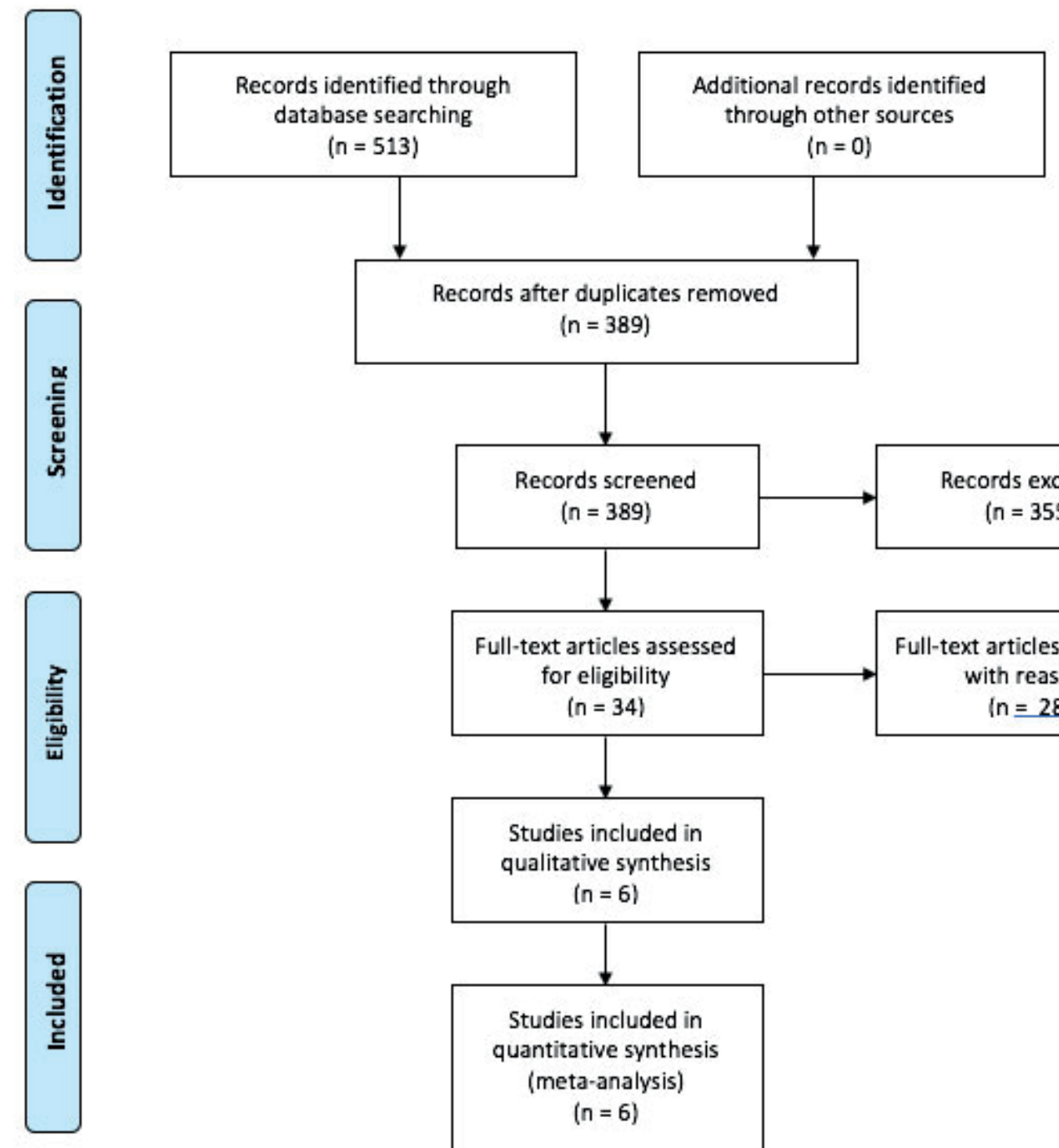

Figure 1. PRISMA chart flow, depicting the studies inclusion process after careful literature review.

\section{Study Quality Assessment}

The quality of the study was assessed with the Modified Coleman Methodology Score (mCMS), represented graphically in table 1. This tool, employed for quality assessment, takes into consideration 10 criteria that address the methodology of the study, giving a value from a minimum of 0 to a maximum of 100 . A score of a 100 signifies a reduced risk of bias and a strong limitation to possible methodological mistakes. The final score can be then stratified based on the following scheme: 100 to 85 points is "Excellent", 85 to 70 points is "Good", 70 to 50 points is "Fair" and < 50 points is "Poor". The subsections of the CMS are based on the CONSORT statement for randomized controlled trials modified to fit also other study designs [10].

\section{Data analysis}

For analyses of direct comparisons between the cemented TKA group and the cementless TKA groups we used Prometa3 version 2.1. For each outcome of interest, in addition to the overall size effect, a heterogeneity analysis was performed. For all tests a $P$ value $<0.05$ indicates statistical significance. Instead for the analysis of the groups demographic characteristics a Pearson correlation test was performed. 


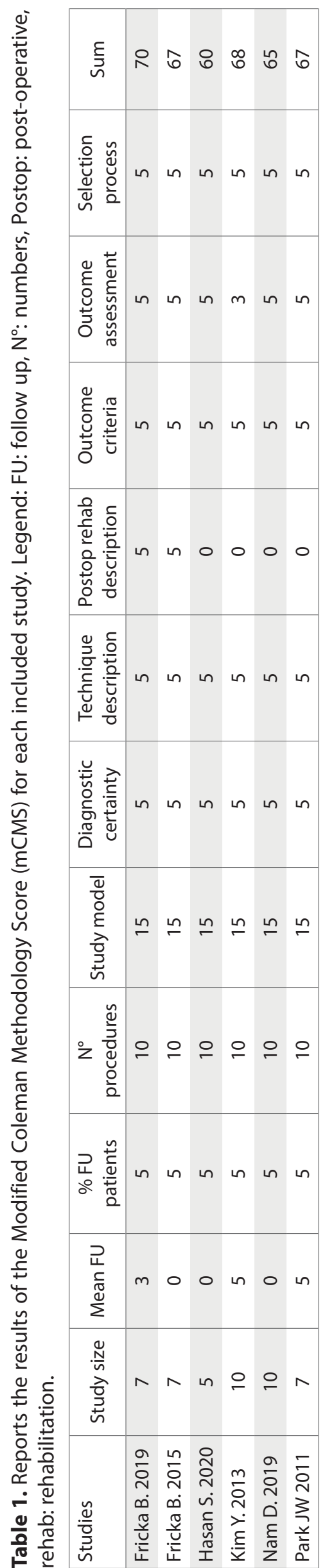

\section{RESULTS}

\section{Search results}

The literature review resulted in 513 studies, but only six [11-16] met the inclusion criteria set and were finally included in the current systematic review. The six included studies were all randomized controlled trials (RCT) with a level of evidence I. They had a mean follow up of 6.9 years (range 2 - 16.6), The quality of the included studies was defined as "Fair" according to the mCMS score (mean points 66.2).

\section{Surgical technique}

Among the 6 included studies, only two studies did not perform the patellar resurfacing during the TKA surgery [13]15]. Concerning the components of implants, in four studies [11][12][14][16] the cementless group received the Zimmer NexGen ${ }^{\circledR}$ cementless femur CR flex design with a fiber metal mesh ingrowth surface and a cementless modular trabecular metal pegged tibia tray while the cemented group received the Zimmer Biomet NexGen ${ }^{\circledR}$ cemented precoat CR flex femur and a cemented precoat keeled tibia tray. Both treatment groups received the same standard CR fixedbearing polyethylene liner. The last two studies employed respectively the Tritanium Triathlon CR TKA (Stryker) [13] and the beaded, Peri-Apatitecoated TKA (Stryker) [15].

\section{Demographics}

The final study group was composed by 521 patients and 651 knees; the discrepancy between these two values is explained by the fact that 130 patients have undergone a bilateral TKA. Among the 651 treated knees, 319 were cemented (cement group) and 332 were not cemented (cementless group). The cemented group population was composed by $44 \%$ of males, had a mean age at surgery of 60 years and average body mass index (BMI) of 29.9. Instead the cementless group population was composed by $46 \%$ of males, had a mean age at surgery of 59.8 years and average body mass index (BMI) of 28.9. The statistical analysis of these demographic values revealed no statistically significant difference as summarized in Table 2.

\section{Clinical Outcomes}

The six studies included in the current review employed the following clinical scores in order 
Table 2. Summarizes the statistical analysis performed on the two groups in order to identify possible differences in the study populations. Legend: BMI: body mass index.

\begin{tabular}{|l|c|c|c|}
\hline Variables & $\begin{array}{c}\text { Cementless } \\
\text { group }\end{array}$ & $\begin{array}{c}\text { Cemented } \\
\text { groups }\end{array}$ & $\begin{array}{c}\text { Statistical } \\
\text { analysis }\end{array}$ \\
\hline Number of knees & 332 & 319 & P value $>0.05$ \\
Number of males & 152 & 140 & P value $>0.05$ \\
Age at surgery & 59.8 & 60 & P value $>0.05$ \\
BMl & 28.9 & 29.9 & P value $>0.05$ \\
\hline
\end{tabular}

to investigate the clinical outcomes of the TKA surgery: Knee Society Score (KSS) clinical and functional, the Oxford Knee Score (OKS) and the Visual Analogue Score (VAS). In particular, all the studies assessed the KSS clinical reporting in the cemented group a net increase of 53.2 points from a baseline of 38.7 to 91.9 at last follow up. Instead in the cementless group a net increase of 52.8 points from a baseline of 38.4 to 91.3 at last follow up was observed. The difference between the two groups was not statistically significant as can be appreciated in Figure 2.

Instead only four studies [11][12][13][16] investigated the KSS functional reporting in the cemented group a net increase of 32.6 points from a baseline of 55.7 to 88.3 at last follow up. Instead in the cementless group a net increase of 33.3 points from a baseline of 54.9 to 88.2 at last follow up was observed. The difference between the two groups was not statistically significant as can be appreciated in Figure 3.
Only three studies [11][12][15] assessed the OKS reporting in the cemented group a net increase of 22.1 points from a baseline of 20.9 to 43 at last follow up. Instead in the cementless group a net increase of 20.3 points from a baseline of 22.1 to 42.4 at last follow up was observed. The difference between the two groups was not statistically significant as can be appreciated in Figure 4.

Three studies [11][12][15] assessed the VAS reporting in the cemented group a net decrease of 2.8 points from a baseline of 5.8 to 3 at last follow up. Instead in the cementless group a net decrease of 2.5 points from a baseline of 5.5 to 3 at last follow up was observed. The difference between the two groups was not statistically significant as can be appreciated in Figure 5.

\section{Other Clinical Parameters}

Three studies [11][12][15] reported the length of surgery, reporting a mean value in the cemented group of 85.2 minutes and of 76.7 minutes in the cementless group. Three studies [14-16] investigated the quantity of blood loss during the surgery, reporting a mean value of blood loss in the cemented group of $1342 \mathrm{ml}$ and of $1509 \mathrm{ml}$ in the cementless group.

\section{Radiological Evaluation}

All the studies reported the number of TKA revision occurred during the follow up. A similar

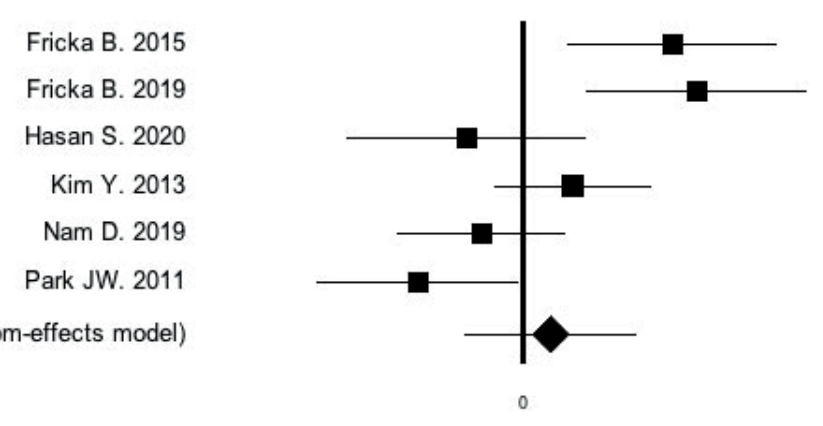

\begin{tabular}{|c|c|c|c|c|c|c|c|c|c|c|c|c|c|c|}
\hline Id & ES & $\mathrm{LL}$ & UL & Sig. & v & SE & w & Res. & R-Sig. & $\mathrm{N}$ & & $\mathrm{n} 1$ & $\mathrm{n} 2$ & $\mathrm{n}$ na \\
\hline Fricka B. 2015 & 0.59 & 0.17 & 1.01 & 0.005 & 0.04 & 0.21 & $16.28 \%$ & 1.29 & 0.197 & & 93 & 46 & 47 & 0 \\
\hline Fricka B. 2019 & 0.69 & 0.25 & 1.12 & 0.002 & 0.05 & 0.22 & $15.85 \%$ & 1.60 & 0.109 & & 85 & 44 & 41 & 0 \\
\hline Hasan S. 2020 & -0.22 & -0.69 & 0.25 & 0.360 & 0.06 & 0.24 & $15.18 \%$ & -0.78 & 0.438 & & 69 & 34 & 35 & 0 \\
\hline Kim Y. 2013 & 0.20 & -0.11 & 0.51 & 0.213 & 0.03 & 0.16 & $18.22 \%$ & 0.20 & 0.839 & & 160 & 80 & 80 & 0 \\
\hline Nam D. 2019 & -0.16 & -0.50 & 0.17 & 0.333 & 0.03 & 0.17 & $17.84 \%$ & -0.68 & 0.496 & & 141 & 65 & 76 & 0 \\
\hline Park JW. 2011 & -0.41 & -0.81 & -0.02 & 0.041 & 0.04 & 0.20 & $16.64 \%$ & -1.46 & 0.146 & & 100 & 50 & 50 & 0 \\
\hline \multicolumn{15}{|l|}{ Overall effect size } \\
\hline Id & $k$ & ES & LL & UL & & ig. & v & SE & $\mathrm{N}$ & & $\mathrm{n} 1$ & n2 & & na \\
\hline Overall (random-effects model) & 6 & 0.11 & -0 . & .23 & 0.45 & 0.529 & 0.03 & 0.17 & & 648 & & 319 & 329 & 0 \\
\hline
\end{tabular}

Figure 2. Shows the statistical analysis concerning the KSS clinical between the 2 groups: cemented group (on the right of the forest plot) and the cementless group (on the left). Legend: Sig: significance, V: variance. 
Fricka B. 2015

Fricka B. 2019

Hasan S. 2020

Park JW. 2011

Overall (random-effects model)

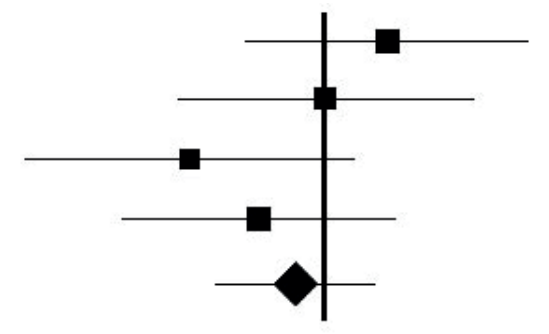

0

\begin{tabular}{|c|c|c|c|c|c|c|c|c|c|c|c|c|c|}
\hline Id & ES & LL & UL & Sig. & v & SE & w & Res. & R-Sig. & $\mathrm{N}$ & $\mathrm{n} 1$ & $\mathrm{n} 2$ & $\mathrm{n}$ na \\
\hline Fricka B. 2015 & 0.18 & -0.22 & 0.59 & 0.375 & 0.04 & 0.21 & $26.59 \%$ & 1.47 & 0.141 & 93 & 46 & 47 & 0 \\
\hline Fricka B. 2019 & 0.01 & -0.42 & 0.43 & 0.969 & 0.05 & 0.22 & $24.75 \%$ & 0.37 & 0.710 & 85 & 44 & 41 & 0 \\
\hline Hasan S. 2020 & -0.38 & -0.86 & 0.09 & 0.115 & 0.06 & 0.24 & $20.45 \%$ & -1.41 & 0.159 & 69 & 34 & 35 & 0 \\
\hline Park JW. 2011 & -0.18 & -0.58 & 0.21 & 0.359 & 0.04 & 0.20 & $28.21 \%$ & -0.45 & 0.653 & 100 & 50 & 50 & 0 \\
\hline
\end{tabular}

Overall effect size

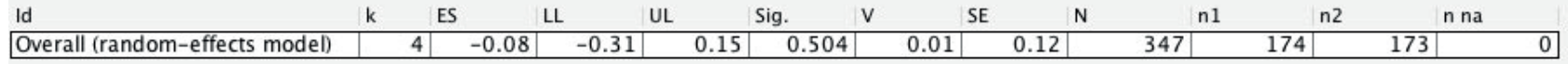

Figure 3. Shows the statistical analysis concerning the KSS functional between the 2 groups: cemented group (on the right of the forest plot) and the cementless group (on the left). Legend: Sig: significance, V: variance.

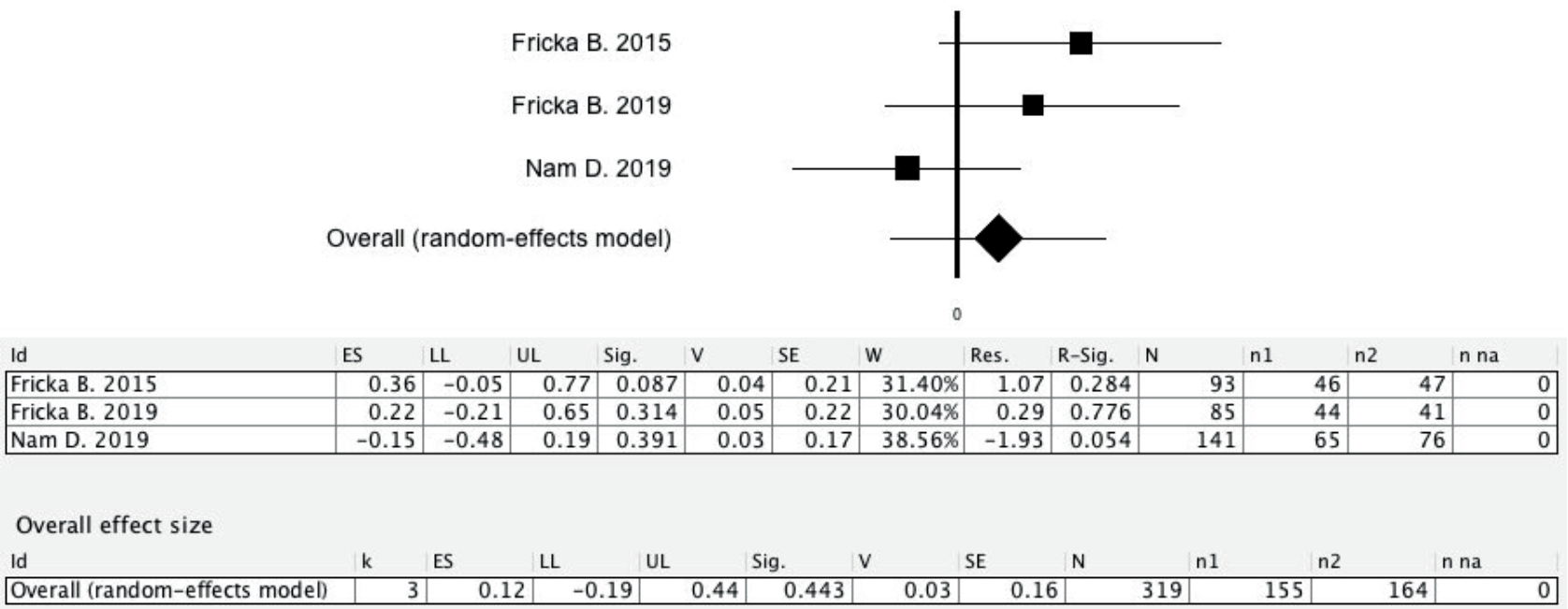

Figure 4. Shows the statistical analysis concerning the OKS functional between the 2 groups: cemented group (on the right of the forest plot) and the cementless group (on the left). Legend: Sig: significance, V: variance.

Fricka 2015
Fricka 2019
Nam D. 2019

Overall (random-effects model)

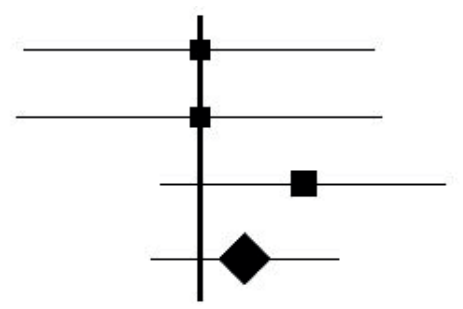

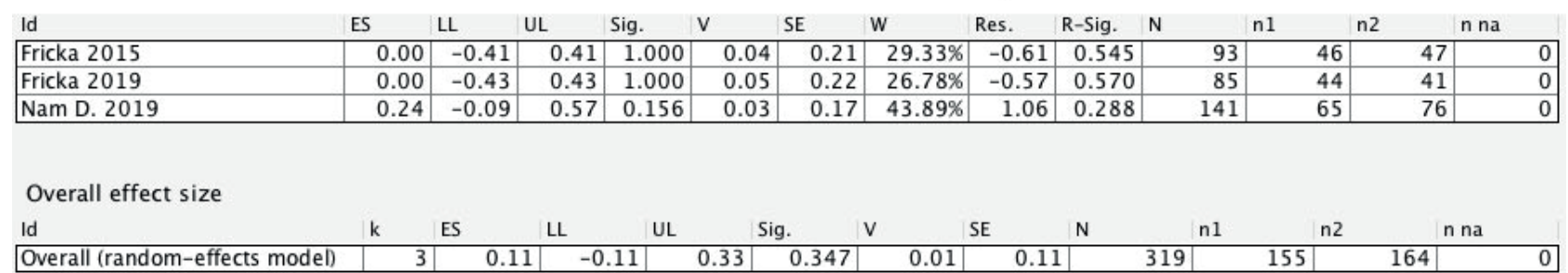

Figure 5. Shows the statistical analysis concerning the VAS functional between the 2 groups: cemented group (on the right of the forest plot) and the cementless group (on the left). Legend: Sig: significance, V: variance. 
number of revisions was observed in both groups with 3 cases in the cemented group and 4 in the cementless group and the difference was not statistically significant ( $p$ value: 0.700 ). Instead four studies [11][12][14][16] reported the presence of radiolucencies, in particular 41 cases in the cemented group and 68 cases in the cementless group, the difference was found to be statistically significant ( $p$ value: 0.007 ). Moreover, only two studies [11-12] reported the rate of subsidence, in particular 0 cases were observed in the cemented group and 8 in the cementless group, the difference was not statistically significant (P value: 1.016).

\section{DISCUSSION}

The main finding of the current systematic review and meta-analysis is the non-inferiority of the cementless TKA implant compared to the cemented TKA in terms of clinical outcomes and complications rate. Indeed, the statistical analysis revealed no significant differences in all clinical scores and a similar revision rate in the two groups making the cementless technique as effective and safe as the cemented one. In the late 90 ' a number of clinical trials and reviews were published observing the inferiority of the cementless technique both in terms of safety (higher complications rate) and efficacy (lower clinical scores improvement) [17, 18]. These discouraging results of cementless TKAs have determined the use of cemented implants worldwide [19]. In the first decade of the 2000 instead, the cemented technique showed an improvement in terms of efficacy, with a metanalysis of 15 clinical studies [2] showing no difference in efficacy (same KSS improvement) between the two groups, but still a higher rate of complications in the cementless group was observed. Eventually in the second decade of the 2000, the rate of improvement of the cementless has kept rising, as observed by the current review of RCTs that showed the non-inferiority of the cemented technique compared to the cemented one not only in terms of clinical scores improvement but also in survival rate. The improved performance of the cementless technique with the decades may be explained by the development of biotechnologies and biomaterials with high osteoconductive properties [19]. The results of the current review are in line with two recent systematic reviews, showing how modern cementless TKA implants employed in the period 2010-2020 showed excellent survival rate and clinical outcomes improvement [20, 21]. Moreover, the cementless technique offers additional advantages compared to the cemented one, considering the epidemiological trends of the TKA surgery. Indeed, the rate of knee joint replacement performed in younger patients has increased together with the rise of humans' life span, leading to a higher rate of implants' revisions in the future [1]. Given the epidemiological context just described, the cementless technique could provide favorable outcomes since guarantees a more biologic fixation of the implant, considering that the porous surface facilitates a greater bone ingrowth, as osteoblasts and mesenchymal cells migrate toward the implant [5] [22].

Another advantage offered by the cementless technique concerns the surgical time. Indeed, a significant mean reduction of the operating time of 8,5 minutes $(9,98 \%)$ was found in the current study when implanting cementless TKAs with respect to the cemented group: the mean surgical time for the cementless group resulted to be 76,7 minutes, while for the cemented group resulted to be 85,2 minutes on average. This is due to the use of cement that is a procedure involving specific steps: preparation, application on joint and implant, waiting for cement curing, removal of residual cement and washing. This procedure has to be followed accurately and so it requires time, ultimately prolonging the stay in the operating room. Given that, the use of cementless TKAs is undeniably timesaving. In addition, the reduced surgical time lowers the time of exposure to pneumatic ischemia and to possible contaminants, bringing about benefits for both the surgeon and the patient [23]. Moreover, the employment of a cementless technique leads to an overall decrease in procedural cost of the TKA surgery of about $\$ 366$ per implant [24].

Despite our metanalysis has discarded this kind of studies, several trials have proposed the technique of the Hybrid Fixation consisting in the cementation of the tibial component only while the femoral component remained cementless. The results showed non-inferiority of the hybrid implant compared to the cemented one, but the follow up used was short and perhaps insufficient to actually infer reliable conclusions in one case ( 2 years) [25] and in the other the heterogeneity of 
implants may have played as a source of bias [26]. Furthermore, with the hybrid fixation the advantage derived from the reduction in surgical time and of pneumatic ischemia, seen in the cementless technique, is lost. Concerning the limitations of the current study, should be mentioned the paucity of included studies (due to the stringent inclusion criteria), the limited mean follow-up time, the heterogeneity of the clinical scores and the possible source of bias given by the patellar resurfacing employed buy some included studies and not by others. In conclusion, the results of the current metanalysis suggest the non-inferiority of cementless fixation with respect to cemented
TKAs in terms of clinical outcomes and survival rates of the implants. Therefore, now potentially advantages of the cementless technique over the cemented one could be investigated both in terms of clinical outcomes and complications rate. Yet in order to discern if these potential advantages are real, studies with a longer follow-up and a better study design are needed.

\section{CONFLICT Of INTEREST STATEMENT}

Authors declare no conflict of interests.
[1] Kurtz SM, Lau E, Ong K, et al. Future Young Patient Demand for Primary and Revision Joint Replacement: National Projections from 2010 to 2030. Clin Orthop Relat Res 2009; 467: 2606-12. https://doi.org/10.1007/s11999-009-0834-6.

[2] Gandhi R, Tsvetkov D, Davey JR, et al. Survival and clinical function of cemented and uncemented prostheses in total knee replacement: a meta-analysis. J Bone Joint Surg Br 2009; 91: 889-95. https://doi.org/10.1302/0301620X.91B7.21702.

[3] Zhou K, Yu H, Li J, Wang H, et al. No difference in implant survivorship and clinical outcomes between fullcementless and full-cemented fixation in primary total knee arthroplasty: A systematic review and meta-analysis. Int J Surg 2018; 53: 312-9. https://doi.org/10.1016/j. ijsu.2018.04.015.

[4] Berger RA, Lyon JH, Jacobs JJ, et al. Problems with cementless total knee arthroplasty at 11 years followup. Clin Orthop Relat Res 2001: 196-207. https://doi. org/10.1097/00003086-200111000-00024.

[5] Karageorgiou V, Kaplan D. Porosity of 3D biomaterial scaffolds and osteogenesis. Biomaterials 2005; 26: 547491. https://doi.org/10.1016/j.biomaterials.2005.02.002.

[6] Henricson A, Linder L, Nilsson KG. A trabecular metal tibial component in total knee replacement in patients younger than 60 years: a two-year radiostereophotogrammetric analysis. J Bone Joint Surg Br 2008; 90: 1585-93. https:// doi.org/10.1302/0301-620X.90B12.20797.

[7] Fernandez-Fairen $M$, Hernández-Vaquero D, Murcia $A$, et al. Trabecular metal in total knee arthroplasty associated with higher knee scores: a randomized controlled trial. Clin Orthop Relat Res 2013; 471: 3543-53. https://doi. org/10.1007/s11999-013-3183-4

[8] Higgins JP, Green S. Cochrane Handbook for Systematic Reviews of Interventions : Cochrane Book Series n.d.:674.

[9] Kendall C, Maskalyk J, Palepu A. Closing Open Medicine. Open Med 2014; 8: e147-9.
[10] Schulz KF, Altman DG, Moher D. CONSORT Group. CONSORT 2010 statement: updated guidelines for reporting parallel group randomised trials. BMJ 2010; 340: c332. https://doi.org/10.1136/bmj.c332.

[11] Fricka KB, McAsey CJ, Sritulanondha S. To Cement or Not? Five-Year Results of a Prospective, Randomized Study Comparing Cemented vs Cementless Total Knee Arthroplasty. J Arthroplasty 2019; 34: S183-7. https://doi. org/10.1016/j.arth.2019.02.024.

[12] Fricka KB, Sritulanondha S, McAsey CJ. To Cement or Not? Two-Year Results of a Prospective, Randomized Study Comparing Cemented Vs. Cementless Total Knee Arthroplasty (TKA). J Arthroplasty 2015; 30: 55-8. https:// doi.org/10.1016/j.arth.2015.04.049.

[13] Hasan S, van Hamersveld KT, Marang-van de Mheen PJ, et al. Migration of a novel 3D-printed cementless versus a cemented total knee arthroplasty: two-year results of a randomized controlled trial using radiostereometric analysis. Bone Joint J 2020; 102-B: 1016-24. https://doi. org/10.1302/0301-620X.102B8.BJJ-2020-0054.R1.

[14] Kim Y-H, Park J-W, Lim H-M, et al. Cementless and cemented total knee arthroplasty in patients younger than fifty five years. Which is better? Int Orthop 2014; 38: 297-303. https://doi.org/10.1007/s00264-013-2243-4.

[15] Nam D, Lawrie CM, Salih R, et al. Cemented Versus Cementless Total Knee Arthroplasty of the Same Modern Design: A Prospective, Randomized Trial. J Bone Joint Surg Am 2019; 101: 1185-92. https://doi.org/10.2106/ JBJS.18.01162.

[16] Park J-W, Kim Y-H. Simultaneous cemented and cementless total knee replacement in the same patients: a prospective comparison of long-term outcomes using an identical design of NexGen prosthesis. J Bone Joint Surg Br 2011; 93 : 1479-86. https://doi.org/10.1302/0301-620X.93B11.27507

[17] Rand JA. Cement or cementless fixation in total knee arthroplasty? Clin Orthop Relat Res 1991: 52-62. 
[18] McCaskie AW, Deehan DJ, Green TP, et al. Randomised, prospective study comparing cemented and cementless total knee replacement: results of press-fit condylar total knee replacement at five years. J Bone Joint Surg Br 1998; 80: 971-5. https://doi.org/10.1302/0301-620x.80b6.8558.

[19] Matassi F, Carulli C, Civinini R, et al. Cemented versus cementless fixation in total knee arthroplasty. Joints 2014; 1: 121-5.

[20] Haeberle HS, Salem HS, Ehiorobo JO, et al. Newer Generation of Cementless Total Knee Arthroplasty: A Systematic Review. Surg Technol Int 2020; 36: 351-9.

[21] Newman JM, Sodhi N, Dekis JC, et al. Survivorship and Functional Outcomes of Cementless versus Cemented Total Knee Arthroplasty: A Meta-Analysis. J Knee Surg 2020; 33: 270-8. https://doi.org/10.1055/s-0039-1678525.

[22] Jones AC, Arns $\mathrm{CH}$, Sheppard AP, et al. Assessment of bone ingrowth into porous biomaterials using MICRO-CT. Biomaterials 2007; 28: 2491-504. https://doi.org/10.1016/j. biomaterials.2007.01.046.
[23] Ejaz A, Laursen AC, Kappel A, et al. Tourniquet induced ischemia and changes in metabolism during TKA: a randomized study using microdialysis. BMC Musculoskelet Disord 2015; 16: 326. https://doi.org/10.1186/s12891-0150784-y.

[24] Lawrie CM, Schwabe M, Pierce A, et al. The cost of implanting a cemented versus cementless total knee arthroplasty. Bone Joint J 2019; 101-B: 61-3. https://doi. org/10.1302/0301-620X.101B7.BJJ-2018-1470.R1.

[25] Gao F, Henricson A, Nilsson KG. Cemented versus uncemented fixation of the femoral component of the NexGen CR total knee replacement in patients younger than 60 years: a prospective randomised controlled RSA study. Knee 2009; 16: 200-6. https://doi.org/10.1016/j. knee.2008.11.009.

[26] Yang J-H, Yoon J-R, Oh C-H, et al. Hybrid component fixation in total knee arthroplasty: minimum of 10-year follow-up study. J Arthroplasty 2012; 27: 1111-8. https:// doi.org/10.1016/j.arth.2011.09.019. 